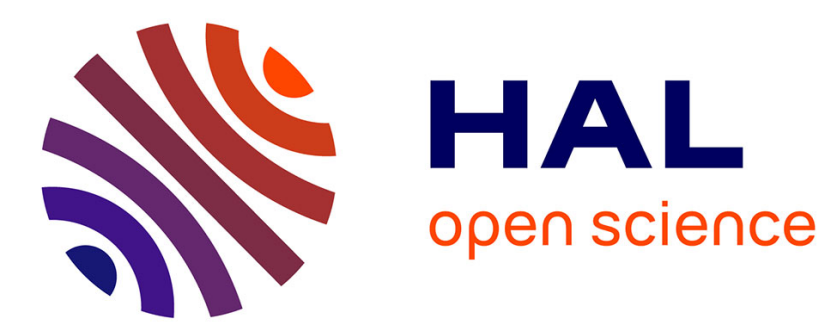

\title{
The destabilization of an initially thick liquid sheet edge
} Henri Lhuissier, Emmanuel Villermaux

\section{- To cite this version:}

Henri Lhuissier, Emmanuel Villermaux. The destabilization of an initially thick liquid sheet edge. Physics of Fluids, 2011, 23 (9), pp.091705. 10.1063/1.3644840 . hal-00714111

\section{HAL Id: hal-00714111 https://hal.science/hal-00714111}

Submitted on 31 Jan 2017

HAL is a multi-disciplinary open access archive for the deposit and dissemination of scientific research documents, whether they are published or not. The documents may come from teaching and research institutions in France or abroad, or from public or private research centers.
L'archive ouverte pluridisciplinaire HAL, est destinée au dépôt et à la diffusion de documents scientifiques de niveau recherche, publiés ou non, émanant des établissements d'enseignement et de recherche français ou étrangers, des laboratoires publics ou privés. 


\title{
The destabilization of an initially thick liquid sheet edge
}

\author{
Henri Lhuissier ${ }^{\text {a) }}$ and Emmanuel Villermaux ${ }^{\text {b) }}$ \\ Aix-Marseille Université, IRPHE, 13384 Marseille Cedex 13, France
}

(Received 30 June 2011; accepted 7 September 2011; published online 30 September 2011)

\begin{abstract}
By forcing the sudden dewetting of a free soap film attached on one edge to a straight solid wire, we study the recession and subsequent destabilization of its free edge. The newly formed rim bordering the sheet is initially thicker than the film to which it is attached, because of the Plateau border preexisting on the wire. The initial condition is thus that of an immobile massive toroidal rim connected to a thin liquid film of thickness $h$. The terminal Taylor-Culick receding velocity $V=\sqrt{2 \sigma / \rho h}$, where $\sigma$ and $\rho$ are the liquid surface tension and density, respectively, is only reached after a transient acceleration period which promotes the rim destabilization. The selected wavelength and associated growth time coincide with those of an inertial instability driven by surface tension. (C) 2011 American Institute of Physics. [doi:10.1063/1.3644840]
\end{abstract}

When a liquid film is punctured, it bursts by opening a hole, and ultimately resolves into a mist of droplets. The hole opening motion is driven by the unbalanced surface tension force $2 \sigma$ at its edge: This force sets into motion the growing mass of liquid collected in the rim, at the constant velocity $V=\sqrt{2 \sigma / \rho h}$ historically determined by Taylor ${ }^{1}$ and Culick. ${ }^{2}$

The subsequent destabilization of the film responsible for its fragmentation may have several causes. For a flat thin soap film punctured in its center, the recession motion is so fast and the film so light that it develops a shear instability with the surrounding environment by flapping like a flag. ${ }^{3}$ When the surface of the liquid film is initially curved, like for a bubble bursting at the surface of a pool, an inertial instability resulting from centrifugal forces induces the rim progressive indentation. ${ }^{4}$ However, in all these situations, the thickness of the rim at the edge of the hole is initially of the order of that of the sheet itself. Here, we present a new situation where the rim is initially much thicker than the sheet to which it is attached. The quantitative exploration of the rim destabilization dynamics offers an opportunity to discuss the nature of the instability mechanisms at play.

Soap films are formed by slowly pulling a vertical frame out of a solution of a commercial surfactant (Dreft by Procter $\&$ Gamble, a mixture of non-ionic and anionic molecules) diluted in tap water. The concentration in surfactant is always larger than the critical micelle concentration above which surface tension remains blocked at its saturation value, which is $\sigma=25 \times 10^{-3} \mathrm{~N} . \mathrm{m}^{-1}$. The frame is a $5 \times 5 \mathrm{~cm}^{2}$ planar square, and is constituted of three rigid aluminum sides forming a reversed $\mathrm{U}$, and of a tighten tungsten wire of diameter $d_{f}=100 \mu \mathrm{m}$ on the fourth bottom side. The wire is electrically isolated from the frame and is connected to a capacitor charged with a high voltage transformer whose discharge can be triggered on demand.

\footnotetext{
${ }^{a)}$ Electronic mail: lhuissier@irphe.univ-mrs.fr.

${ }^{b}$ Electronic mail: villermaux@irphe.univ-mrs.fr. Also at Institut Universitaire de France.
}

Once formed, the vertical soap film is therefore attached on three sides to the rigid frame and on the bottom side to the wire via a meniscus, or Plateau border. The film is left draining until the desired thickness $h$, ranging from a few to tens of a micrometer $(3-30 \mu \mathrm{m})$, is reached. Then the capacitor is discharged through the wire, which instantaneously heats by Joule effect. The discharge provokes the vaporization of part of the liquid in the Plateau border, and the simultaneous detachment of the film all along the wire, as seen in Fig. 1. The precise detachment mechanism probably also involves the impulse communicated to the Plateau border as the wire suddenly dilates. However, its detail is unimportant; it realizes the required initial configuration of an almost straight free liquid film edge with a massive rim, whose velocity is small with respect to the Culick-Taylor velocity $V$.

The rim is visualized by light refraction from a uniform back-lighting with a Photron APX fast camera operating at frame rates between 5000 and $15000 \mathrm{~s}^{-1}$, and synchronized with the capacitor discharge. Immediately after detachment from the wire, the film recedes and the initially straight rim corrugates. Corrugations amplitudes regularly grow for a transient period of a few milliseconds, until they form regularly spaced deep indentations, which further coarsen by eikonal propagation, ultimately forming cusps ${ }^{5}$ at the tip of which drops are released. We do not consider here this latter long time dynamics and focus on the early transient period of the instability.

Denoting $z$ the coordinate along the wire and $\xi \equiv \xi(z, t)$ the distance normal to the wire in the $x$ direction towards the film edge (Fig. 1), we define its mean position $\langle\xi\rangle_{z}$. A typical time evolution of $\langle\xi\rangle_{z}$ is shown in Fig. 2. The receding velocity is clearly not constant, and the rim is continuously accelerated. This is not a slow viscous transient, ${ }^{6-8}$ since the viscous characteristic time $h \eta / \sigma$ is negligible in comparison with the inertial characteristic time $\sqrt{\rho h^{3} / \sigma}$. This is rather a consequence of the large initial inertia of the rim, which must be absorbed before the terminal velocity $V$ is reached. Indeed, as seen in Fig. 1, the Plateau border has a cross section much larger than $h^{2}$, and as the film edge detaches, it 


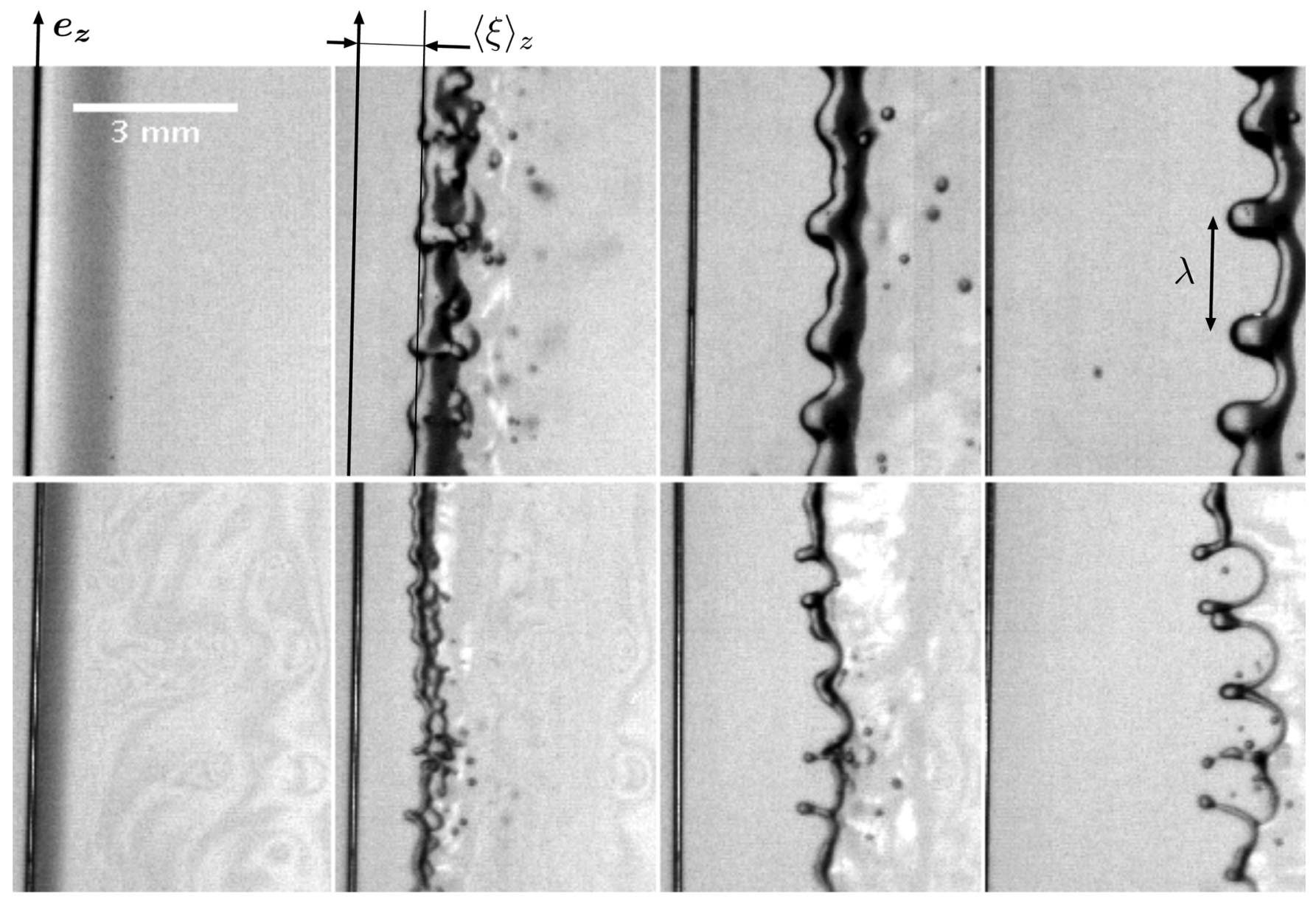

FIG. 1. Destabilization of the initially straight edge of two receding soap films having distinct thicknesses $h$, and rim mass $\rho h x_{0}$ (defined in Eq. (1) and both measured by fitting the rim trajectory to Eq. (2) as in Fig. 2). Successive images are separated by $2.4 \mathrm{~ms}$ and $0.8 \mathrm{~ms}$ on the top (for which $x_{0} / h=2604$ ) and bottom sequence (with $x_{0} / h=424$ ), respectively, and the spatial scale is the same for both (images have been rotated by $90^{\circ}$ clockwise, recession being directed upward and gravity pointing downward in the experiment).

carries with it most of the liquid constitutive of the Plateau border in its rim.

The base state of the rim trajectory can be easily determined considering a film of uniform thickness $h$, invariant with $z$ and whose initial conditions are known. Disregarding the details of the rim shape and assimilating it to a punctual mass, the receding dynamics is obtained from global mass and momentum balances

$$
\frac{\mathrm{d}}{\mathrm{d} t}\left(\rho h x \frac{\mathrm{d} x}{\mathrm{~d} t}\right)=2 \sigma, \quad x(0)=x_{0}, \quad \frac{\mathrm{d} x}{\mathrm{~d} t}(0)=V_{0},
$$

where $x$ is the position of the rim center of mass (which will be identified with $\left.x_{0}+\langle\xi\rangle_{z}\right), x_{0}$ is defined from its initial mass $\rho h x_{0}$ and $V_{0}$ is its initial velocity. The solution for Eq. (1) is

$$
x=\sqrt{x_{0}^{2}+2 x_{0} V_{0} t+V^{2} t^{2}} \quad \text { with } \quad V=\sqrt{\frac{2 \sigma}{\rho h}} .
$$

The maximal acceleration the rim experiences is the initial acceleration

$$
\gamma_{0}=\left(V^{2}-V_{0}^{2}\right) / x_{0}
$$

typically lasting for $x_{0} / V$ when $V_{0} \neq V$. When a film spontaneously bursts or when it is punctured with a small object such that the rim has a diameter comparable with $h, V_{0} \ll V$ and the receding velocity is reached within the capillary time $\sqrt{\rho h^{3} / \sigma}$ which is very short in practice.

Note that in the calculation above we have approximated the rim as punctual, with an extension considered as small compared to the distance $x-x_{0}$ it has travelled. This condition is valid for a pure liquid of constant surface tension, but it is not for a solution of highly concentrated surfactant. In that case, the surfactant elastic dynamics has to be accounted for, ${ }^{9,10}$ and the rim adopts an extended shape. The true recession dynamics is slightly more complex than the simple version we have adopted in Eqs. (1) and (2), which are however a sufficient approximation for the present discussion. Relative modifications to the receding velocity due to the rim extension are typically of order $1 \%,{ }^{11}$ explaining why the Taylor-Culick prediction has indeed been successfully checked experimentally on soap films. ${ }^{9}$

As seen in Fig. 2, the rim trajectory is actually well described by Eq. (2). The initial mass $\rho h x_{0}$, initial velocity $V_{0}$ and terminal velocity $V$ (and therefore the film thickness $h$ ) can thus be all measured from the rim trajectory. It is typically found that $V_{0} \ll V$ and that $x_{0} / h$ ranges from $10^{2}$ to $10^{3}$. 

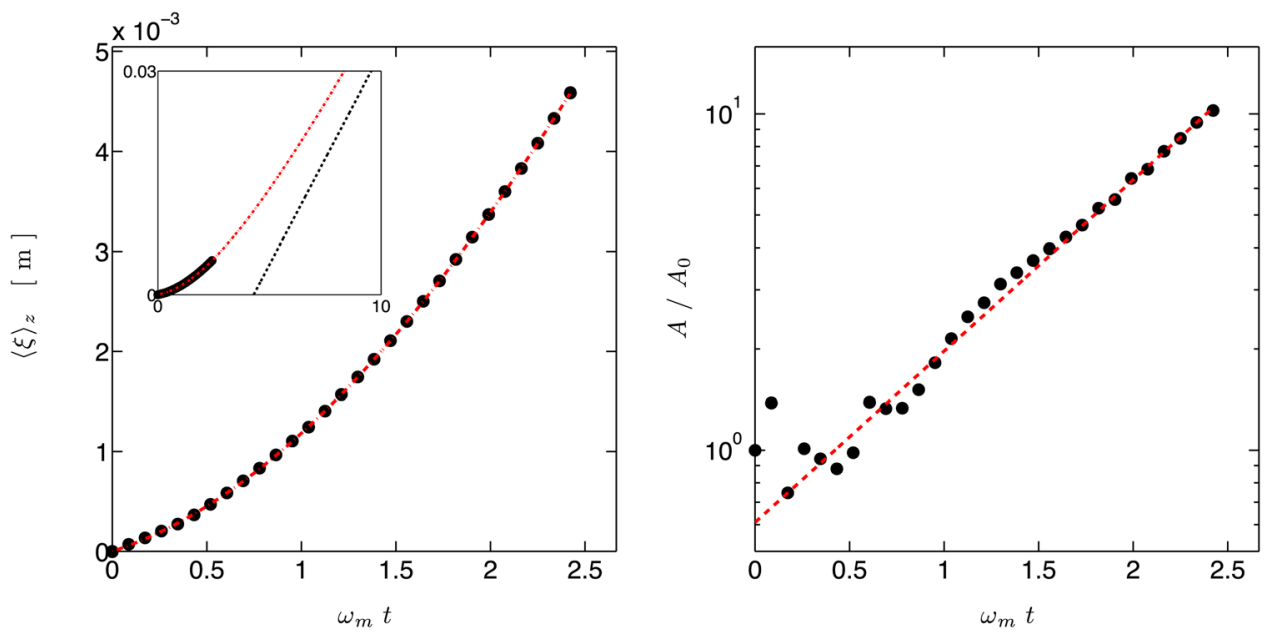

FIG. 2. (Color online) Left: A typical edge position time evolution $(\bullet)$ and its fit by Eq. (2) (--). Inset: Same graph on a longer timescale to show the long time asymptotics $\sim V t(--)$. Right: Time evolution of the corrugations amplitude $A=\sqrt{\left\langle\left(\xi-\langle\xi\rangle_{z}\right)^{2}\right\rangle_{z}}$ for the same liquid film as on the left figure $(\bullet)$ and fit $(--)$ by an exponential growth $\propto \mathrm{e}^{t / \tau}$.

In the same way we defined the mean position of the rim $\langle\xi\rangle_{z}$, the root mean squared corrugations amplitude $A=\sqrt{\left\langle\left(\xi-\langle\xi\rangle_{z}\right)^{2}\right\rangle_{z}}$ is measured for successive times, as seen in Fig. 2. Except for the very short times sensitive to the edge detachment perturbations, the amplitude $A$ grows exponentially at a rate $\tau^{-1}$.

These corrugations are a consequence of the recession dynamics, we have described above. Being continuously accelerated, the rim does not sit in a Galilean frame of reference. A net force is thus imparted to the liquid stored in the rim, which might therefore destabilize. An interface separating two semi-infinite media accelerated at a constant acceleration $\gamma_{0}$ pointing towards the denser phase is unstable in the sense of Rayleigh-Taylor, with a maximal growth rate $\omega_{m}$ and associated most amplified wavelength $\lambda_{m}$

$$
\omega_{m}=\left(\frac{4}{27} \frac{\rho \gamma_{0}^{3}}{\sigma}\right)^{1 / 4} \quad \lambda_{m}=2 \pi \sqrt{\frac{3 \sigma}{\rho \gamma_{0}}} .
$$

Our measurements indicate that the initial velocity $V_{0}$ is always small compared to $V$. The acceleration is thus $\gamma_{0} \simeq V^{2} / x_{0}$ and $\omega_{m}$ amounts to

$$
\omega_{m} \sim\left(\frac{\rho V^{6}}{\sigma x_{0}^{3}}\right)^{1 / 4} \sim \frac{V}{x_{0}}\left(\frac{x_{0}}{h}\right)^{1 / 4} .
$$

As long as the rim is initially thick, that is $x_{0} \gg h$ as for the present case $\left(x_{0} / h=\mathcal{O}\left(10^{2}-10^{3}\right)\right)$, the time of growth of the instability is short compared to the acceleration permanence time $x_{0} / V$, and corrugations have time to grow, driven by an essentially constant acceleration $\gamma_{0}$.

A comparison of the expected growth rate and wavelength in Eq. (4) with the measured growth rates $\tau^{-1}$ and wavelengths $\lambda$ for different films with different thicknesses $h$, initial mass $\rho h x_{0}$ and thus different rim accelerations $\gamma_{0}$ is made in Fig. 3.

The symmetry breaking apparent on the distorted rim (on can tell the direction of the acceleration by looking at still pictures) where thicker portions of the rim are "left behind" thinner ones while accelerated at constant force, ${ }^{12}$ together with the fair quantitative agreement between the observations and Eq. (4) both tend to support the relevance of the Rayleigh-Taylor scenario: The driving force is inertia (acceleration), and the mode selection is made by capillarity. However, the rim is initially thick compared to the sheet to which it is attached, and has an essentially cylindrical shape; one could thus equally invoke a mechanism where surface tension is the driving force of the instability, and inertia is just slowing it down: The Plateau-Rayleigh scenario.

That duality is not new, and has been discussed at length in several related contexts, like to describe the indentations of the liquid sheet resulting from the impact of two jets, ${ }^{13,14}$ or to understand the Worthington crowns in the impact of a drop on a solid, a liquid layer or in a fast gas stream, ${ }^{15-17}$ and for more general straight receding free edges. ${ }^{18-20}$

The present academic study offers the possibility to address this issue; the answer is, however, equally disappointing, and straightforward: In the limit of a massive rim

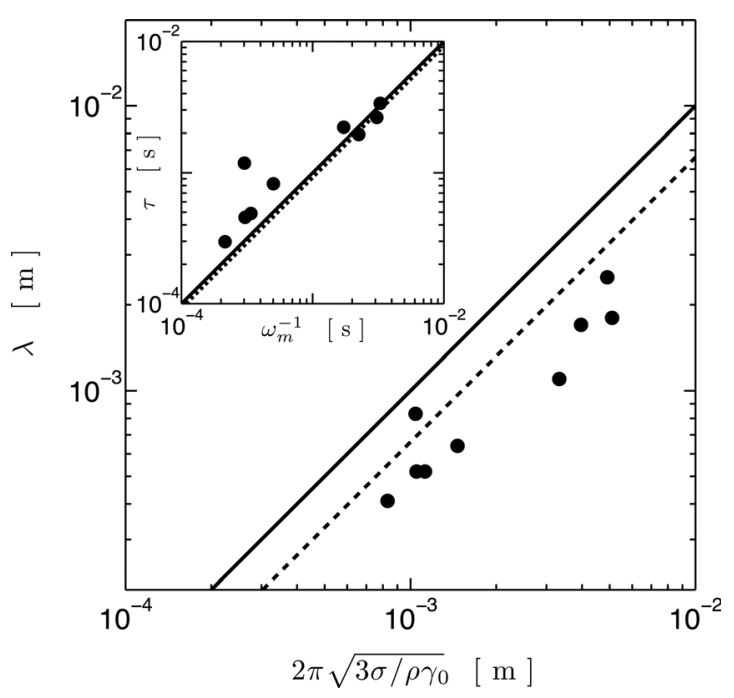

FIG. 3. Wavelength $\lambda$ for various initial rim accelerations $\gamma_{0}$ as measured on images like in Fig. 1 versus the prediction of Eq. (4) (-), and according to the Plateau-Rayleigh scenario with $\lambda_{\mathrm{PR}}=2 \pi / 0.70 r_{0} \simeq 0.66 \lambda_{m}$ with $h x_{0}=\pi r_{0}^{2}(--)$. Inset: Instability growth time $\tau$ as measured on Fig. 2 versus $\omega_{m}^{-1}$ expected from Eq. (4) (-), and according to the Plateau Rayleigh scenario with $\omega_{\mathrm{PR}}=0.34 \sqrt{\sigma / \rho r_{0}^{3}} \simeq 1.09 \omega_{m}(--)$. 
initially at rest (i.e., $x_{0} / h \gg 1$ and $V_{0} / V \ll 1$ ) the film thickness $h$ is not anymore a relevant variable in the short time problem. Viewing the rim as a cylinder of radius $r_{0}$ with linear mass $\rho \pi r_{0}^{2}$, the physical quantities involved are thus limited to $\rho, \sigma$, and $r_{0}$ from which a single time $\tau$ and a single length $\lambda$ can be built, that is to say,

$$
\tau^{-1} \sim \sqrt{\frac{\sigma}{\rho r_{0}^{3}}} \quad \lambda \sim r_{0} .
$$

One recognizes the scaling laws characteristic of the Plateau-Rayleigh destabilization, ${ }^{21}$ which turn out to be also those of the Rayleigh-Taylor destabilization in Eq. (4) if one remembers that $\gamma_{0}=V^{2} / x_{0}$ and $h x_{0}=\pi r_{0}^{2}$. Fig. 3 shows that it is not only illusory to distinguish between the two scenarii in law, but also in absolute value.

In fact both mechanisms are driven by surface tension: the acceleration of the rim is the result of the unbalanced tension force at the film edge. It is then not surprising that the experimental observations are in agreement with the concordant predictions from these two idealized mechanisms which are, at least in the present case, intrinsically undistinguishable.

This work has been supported by the Office national d'études et recherches aérospatiales (ONERA) under contract F/20215/DAT-PPUJ and Agence Nationale de la Recherche (ANR) through Grant ANR-05-BLAN-0222-01. We thank Jérôme Duplat for having judiciously suggested to us the film detachment method.

${ }^{1}$ G. I. Taylor, "The dynamics of thin sheets of fluid III. Desintegration of fluid sheets," Proc. R. Soc. London A 253, 313 (1959).

${ }^{2}$ F. E. C. Culick, "Comments on a ruptured soap film," J. Appl. Phys. 31, 1128 (1960).
${ }^{3}$ H. Lhuissier and E. Villermaux, "Soap films burst like flapping flags," Phys. Rev. Lett. 103, 054501 (2009).

${ }^{4}$ H. Lhuissier and E. Villermaux, "Bursting bubbles," Phys. Fluids 21, 091111 (2009).

${ }^{5}$ A. L. Yarin and D. A. Weiss, "Impact of drops on solid surfaces: Selfsimilar capillary waves, and splashing as a new type of kinematic discontinuity," J. Fluid Mech. 283, 141 (1995).

${ }^{6}$ G. Debrégeas, P. Martin, and F. Brochard-Wyart, "Viscous bursting of suspended films," Phys. Rev. Lett. 75, 3886 (1995).

${ }^{7}$ M. Brenner and D. Gueyffier, "On the bursting of viscous films," Phys. Fluids 11, 737 (1999).

${ }^{8}$ N. Savva and J. W. Bush, "Viscous sheet retraction," J. Fluid Mech. 626, 211 (2009).

${ }^{9}$ W. R. McEntee and K. Mysels, "The bursting of soap films. I. An experimental study," J. Phys. Chem. 73, 3018 (1969).

${ }^{10} \mathrm{H}$. Lhuissier and E. Villermaux, "Destabilization of flapping sheets: The surprising analogue of soap films," C. R. Mécanique, 337, 469 (2009).

${ }^{11} \mathrm{~K}$. Mysels and B. R. Vijayendran, "The bursting of soap films. VIII. Rim velocity in radial bursting," J. Phys. Chem. 81, 731 (1977).

${ }^{12}$ A. Rozhkov, B. Prunet-Foch, and M. Vignes-Adler, "Dynamics of a liquid lamella resulting from the impact of a water drop on a small target," Proc. R. Soc. London A 460, 2681 (2004).

${ }^{13}$ J. W. Bush and A. E. Hasha, "On the collision of laminar jets: fluid chains and fishbones,” J. Fluid Mech. 511, 285 (2004).

${ }^{14} \mathrm{~N}$. Bremond and E. Villermaux, "Atomization by jet impact," J. Fluid Mech. 549, 273 (2006).

${ }^{15}$ L. Zhang, P. Brunet, J. Eggers, and R. Deegan, "Wavelength selection in the crown splash," Phys. Fluids 22 (2010).

${ }^{16} \mathrm{E}$. Villermaux and B. Bossa, "Single drop fragmentation determines size distribution of raindrops," Nature Phys. 5, 697 (2009).

${ }^{17}$ E. Villermaux and B. Bossa, "Drop fragmentation on impact," J. Fluid Mech. 668, 412 (2011).

${ }^{18}$ J. M. Fullana and S. Zaleski, "Stability of a growing end rim in a liquid sheet of uniform thickness," Phys. Fluids 11, 952 (1999).

${ }^{19}$ I. V. Roisman, "On the instability of a free viscous rim," J. Fluid Mech. 661, 206 (2010).

${ }^{20}$ R. Krechetnikov, "Stability of liquid sheet edges," Phys. Fluids 22, 092101 (2010).

${ }^{21}$ J. W. Lord Rayleigh, "On the instability of jets," Proc. R. Soc. London A 10, 4 (1878). 